\title{
microRNA-214 enhances the invasion ability of breast cancer cells by targeting p53
}

\author{
FANG WANG ${ }^{1}$, PENGWEI LV ${ }^{1}$, XINWEI LIU ${ }^{1}$, MINGZHI ZHU ${ }^{1}$ and XINGUANG QIU ${ }^{2}$ \\ ${ }^{1}$ Second Department of Breast Surgery, and ${ }^{2}$ Department of Thyroid Surgery, \\ The First Affiliated Hospital of Zhengzhou University, Zhengzhou, Henan 450052, P.R. China
}

Received December 17, 2014; Accepted February 12, 2015

DOI: $10.3892 /$ ijmm.2015.2123

\begin{abstract}
Breast cancer (BC) is the foremost cause of cancerrelated mortality in women worldwide. An increasing number of studies has confirmed that microRNAs (miRNAs or miRs) play an important role in the development and progression of BC. microRNA-214 (miR-214), a member of the miRNA family, has been demonstrated to function as both a tumor suppressor and oncogene in various types of human cancer. However, the biological function of miR-214 in BC remains unclear. The present study was designed to investigate the potential role of miR-214 in the development and progression of BC. Our results revealed that miR-214 expression was significantly increased in the BC tissues compared with the adjacent benign tissues, and that the upregulation of miR-214 was significantly associated with the invasion ability of the BC cells. Furthermore, $\mathrm{p} 53$, which has been reported to be downregulated in BC, was predicted to be the target gene of miR-214 using bioinformatics software programs. Moreover, luciferase reporter vectors were constructed and it was confirmed that p53 is a target of miR-214. Following the transfection of miR-214 into BC cells, we found that the overexpression of miR-214 markedly enhanced cell invasion through the downregulation of p53 expression. By contrast, the overexpression of p53 abrogated the effects of miR-214. In conclusion, this study demonstrates that miR-214 functions as an oncogene in BC, at least partly by promoting cell invasion through the downregulation of $\mathrm{p} 53$. Therefore, miR-214 may be a potential therapeutic target for the treatment of $\mathrm{BC}$.
\end{abstract}

Correspondence to: Dr Xinguang Qiu, Department of Thyroid Surgery, The First Affiliated Hospital of Zhengzhou University, 1 Jianshe East Road, Zhengzhou, Henan 450052, P.R. China E-mail: xinguangqiuhn@163.com

Abbreviations: BC, breast cancer; miRNAs, microRNAs; MERs, miRNA recognition elements; miR-214, microRNA-214; RT-qPCR, reverse transcription-quantitative polymerase chain reaction; MMPs, matrix metalloproteinases; ECM, extracellular matrix; TIMPs, tissue inhibitors of matrix metalloproteinases

Key words: breast cancer, microRNA-214, cell invasion, p53

\section{Introduction}

Breast cancer (BC) is the most common type of cancer among women worldwide and is driven by multiple genetic or epigenetic alterations and molecular events (1). In spite of various existing screening programs and novel therapeutic strategies that have been applied in the treatment of $\mathrm{BC}$, thus significantly reducing the mortality rates, the molecular mechanisms underlying the pathogenesis of BC are only partially understood (2). In the past, when studying the mechanism of one specific gene, researchers focused on the coding region of the gene. Nowadays, however, the role of non-coding regions has become increasingly important in the investigation of the function of genes.

microRNAs (miRNAs or miRs) are endogenous, non-coding RNAs consisting of 21-23 nucleotides. miRNAs regulate gene expression by binding to miRNA recognition elements (MERs) located in the 3'-untranslated region (3'-UTR) of target messenger RNAs (mRNAs), leading to their translational repression or degradation (3). It has been estimated that $\sim 60 \%$ of all proteincoding genes in humans are regulated by miRNAs (4). Recently, the abnormal expression of miRNAs in several types of cancer has been confirmed, including colon cancer, hepatocellular carcinoma, lung cancer and breast cancer (5-8). Emerging evidence has indicated that miRNAs function as oncogenes or tumor suppressor genes to control cell proliferation, invasion, migration, differentiation and apoptosis (9-11). For example, miR-638 has been shown to inhibit cell proliferation and invasion and to regulate the cell cycle by targeting tetraspanin 1 (12). miR-874 has been shown to inhibit cell proliferation and induce apoptosis by targeting CDK9 (13). miR-215 functions as a prognostic predictor for breast cancer with its high expression in cancer tissues and its association with other clinicopathological factors and the survival of patients (14). These data suggest an important role for miRNAs in the biology of BC.

It has been previously reported that microRNA-214 (miR-214) is dysregulated in several types of human malignancies and plays a variety of roles in tumor progression (15-17). For example, miR-214 has been shown to be upregulated in ovarian cancer (18), gastric cancer (19), Sézary syndrome (20) and melanoma (15), while it has been shown to be downregulated in cervical cancer (21), pancreatic cancer (22) and hepatocellular carcinoma (23). A recent study found that the concentrations of miR-214 were significantly higher in the serum of breast cancer patients in contrast to healthy women (24). However, little is 
known about the association between miR-214 expression levels and the invasion of BC cells.

In the present study, we investigated the potential role of miR-214 in the development and progression of BC. Using reverse transcription-quantitative polymerase chain reaction (RT-qPCR), we observed that miR-214 was markedly upregulated in the $\mathrm{BC}$ tissues compared with the adjacent benign tissues. Functional assays revealed that the overexpression of miR-214 in the BC cells regulated the invasion ability. Furthermore, using the luciferase reporter system, we demonstrated that p53 is a direct target of miR-214. Taken together, our results suggest that miR-214 plays an important role in BC and provide novel insight into the diagnosis and prognosis of $\mathrm{BC}$.

\section{Materials and methods}

Patients. A total of 35 female patients (aged 34-65 years; mean age, 49 years) diagnosed with BC who underwent surgery (underwent mastectomy) were consecutively included in this study. The patients with BC were recruited from the Second Department of Breast Surgery of the First Affiliated Hospital of Zhengzhou University, Zhengzhou, China. All 35 resected BC tissues and corresponding non-malignant breast tissue specimens were collected and immediately frozen in liquid nitrogen and stored in a freezer at $-80^{\circ} \mathrm{C}$. This study was approved by the Ethics Committee of our institution. Written informed consent was provided by all the participants.

miRNA target prediction. The predicted target gene of miR-214 was obtained using an open access database (miRBase, http:// www.mirbase.org/) and three online bioinformatics software programs (RegRNA, http://regrna2.mbc.nctu.edu.tw/; TargetScan, http://www.targetscan.org/; RNA22, http://cbcsrv. watson.ibm.com/rna22.html). The genes co-identified by three programs were considered as potential target genes for miR-214.

$R T-q P C R$. TotalRNA was extracted from the frozen BC tissues and corresponding non-malignant breast tissues using TRIzol reagent (Invitrogen, Carlsbad, CA, USA) according to the manufacturer's instructions. The purity and concentration of the total RNA were determined using an Ultraviolet Spectrophotometer (Eppendorf, Hamburg, German). cDNA was synthesized using the One Step PrimeScript miRNA cDNA synthesis kit (Takara Biotechnology, Dalian, China). Subsequently, mRNA expression was analyzed by quantitative PCR using primers synthesized by the Shanghai Sangon Biological Engineering Technology \& Services Co., Ltd. (Shanghai, China). Briefly, $20 \mu \mathrm{l}$ reactions containing $50 \mathrm{ng}$ of total RNA, $10 \mu \mathrm{l}$ of $2 \mathrm{X}$ SYBR-Green PCR Master Mix, 6.25 U of AMV reverse transcriptase, $10 \mathrm{U}$ of RNase inhibitor and $0.1 \mathrm{mM}$ of primers were subjected to 1 cycle of $95^{\circ} \mathrm{C}$ for $10 \mathrm{~min}$, and then 40 cycles of $95^{\circ} \mathrm{C}$ for $15 \mathrm{sec}, 56^{\circ} \mathrm{C}$ for $30 \mathrm{sec}$ and $72^{\circ} \mathrm{C}$ for $45 \mathrm{sec}$. miR-214 expression was normalized to U6 RNA. Gene mRNA expression was normalized to $\beta$-actin. Data were analyzed using the $\Delta \mathrm{Ct}$ method and expressed as the fold change.

Cell culture and transfection. The human breast cancer cell line, MCF-7, was obtained from the American Type Culture Collection (ATCC; Manassas, VA, USA). The MCF-7 cells were cultured in RPMI-1640 medium (Gibco, Rockville, MD, USA) containing $10 \%$ fetal bovine serum (FBS). For transfec- tion, the hsa-miR-214 mimic, hsa-miR-214 inhibitor, miR-214 mimic control or miR-214 inhibitor control (Ambion, Austin, TX, USA) were delivered at a final concentration of $100 \mathrm{nM}$ using Lipofectamine 2000 reagent (Invitrogen).

Cell invasion assay. The invasion ability of the BC cells was analyzed using Transwell cell culture chambers $(8 \mu \mathrm{m}$ pore size; BD Biosciences, San Jose, CA, USA). Briefly, $48 \mathrm{~h}$ following transfection, the cells were resuspended with serumfree medium, and $200 \mu \mathrm{l}$ of the cell suspension were added to the upper chamber. In addition, medium containing $10 \%$ serum was added to the bottom wells of the 24 -well chamber. The cells were removed from the upper part of the filters by scrubbing with a cotton swab following culture for $24 \mathrm{~h}\left(37^{\circ} \mathrm{C}, 5 \% \mathrm{CO}_{2}\right)$. Subsequently, the membrane was fixed with $4 \%$ formaldehyde for $10 \mathrm{~min}$ at room temperature and stained with $0.5 \%$ crystal violet for $15 \mathrm{~min}$. Finally, the number of invading cells was counted at x100 magnification.

Dual-luciferase reporter assay. A 300 bp sequence from the 3'-UTR of p53 containing a putative miR-214 binding site was amplified by PCR using the cDNA of human genomic DNA as a template. The sequence for the mutation within the miR-214 binding site was amplified by the point mutation method using the KOD-Plus mutagenesis kit (Toyobo, Osaka, Japan) according to the manufacturer's instructions. After subcloning into the pGL3 Luciferase reporter vector (Promega, Madison, WI, USA)

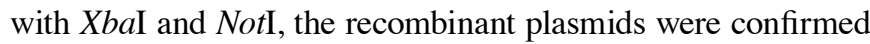
by DNA sequencing. In addition, the recombinant plasmids and $100 \mathrm{nM}$ hsa-miR-214 mimics or the miR-214 mimic control (Ambion, Austin, TX, USA) were transfected into the BC cells using Lipofectamine 2000 for $48 \mathrm{~h}$. Firefly luciferase and Renilla luciferase activity was detected using a fluorescence detector (Promega), and the relative luciferase activity was normalized to Renilla luciferase activity for each transfected well.

Construction and transfection of p53 overexpression vector. Recombinant p53 vectors were constructed using the pFLAG plasmid (Invitrogen). Briefly, the expression sequences encoding p53 were amplified from cDNA, which was synthesized from total RNA extracted from the frozen BC tissues, subcloned into the pFLAG plasmid and then co-transfected with the miR-214 mimic into MCF-7 cells. The negative control group was transfected with the empty pFLAG plasmid and miR-214 mimic control.

Western blot analysis. Equal amounts of protein from the BC cells were denatured at $100^{\circ} \mathrm{C}$ for $5 \mathrm{~min}$, isolated with phosphatebuffered saline (PBS) and separated by 10 or $15 \%$ sodium dodecyl sulfate/polyacrylamide gel electrophoresis (SDS/PAGE). After being transferred onto nitrocellulose membranes and blocked in Tris-buffered saline and Tween buffer containing 5\% non-fat dry milk for $30 \mathrm{~min}$, the target proteins were incubated overnight at $4^{\circ} \mathrm{C}$ with p53 (sc-1311-R), tissue inhibitor of matrix metalloproteinase (TIMP)1 (sc-5538), matrix metalloproteinase (MMP)2 (sc-10736), MMP9 (sc-10737) or $\beta$-actin rabbit anti-human antibodies (sc-10731; Santa Cruz Biotechnology, Inc., Santa Cruz, CA, USA). The membranes were incubated with the corresponding goat anti-rabbit secondary antibodies (sc-45101) for $1 \mathrm{~h}$ at room temperature. A fluorescent western blotting detec- 


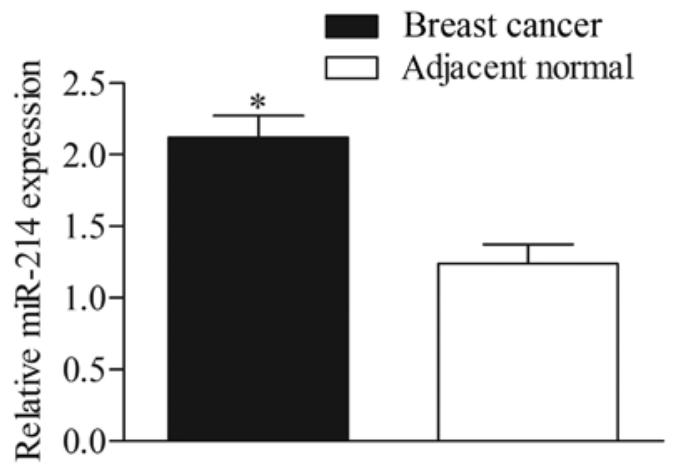

Figure 1. Expression of miR-214 in breast cancer (BC) tissue samples. The expression levels of miR-214 in $35 \mathrm{BC}$ and corresponding non-malignant breast tissues were measured by RT-qPCR. U6 snRNA was used as an internal control for miR-214. Data are represented as the means $\pm S D(n=35)$. ${ }^{*} P<0.05$ vs. adjacent normal group.

tion system was used. The protein bands were quantified using Image-Pro Plus 6.0 and normalized to $\beta$-actin.

Statistical analysis. All data were processed using SPSS 13.0 software. Measurement data are presented as the means \pm standard deviation (SD). Differences among groups were compared using standard deviation followed by an independent-samples t-test. A value of $\mathrm{P}<0.05$ was considered to indicate a statistically significant difference.
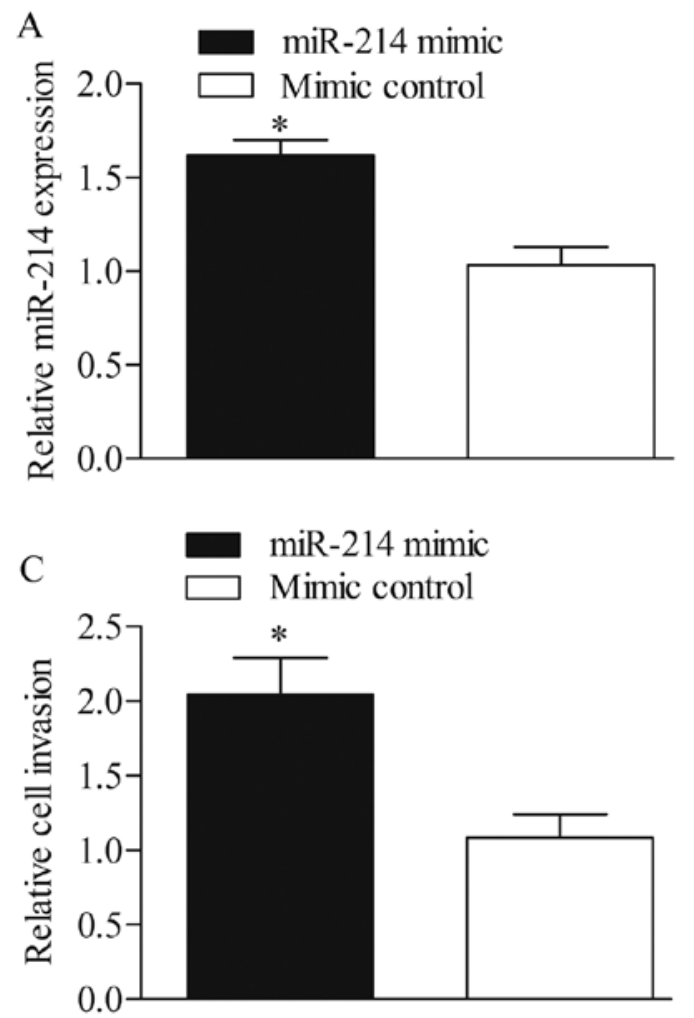

\section{Results}

Increased expression of miR-214 in human BC tissues. The expression levels of mature miR-214 in the BC tissues and corresponding adjacent benign breast tissues were measured by RT-qPCR. The results revelaed that the expression of miR-214 was significantly increased in the BC tissues compared with the adjacent benign breast tissues (Fig. 1).

miR-214 regulates the invasion ability of $B C$ cells. The miR-214 mimic and miR-214 inhibitor were transfected into the MCF-7 cells in order to explore the effects of miR-214 on the invasion ability of BC cells. At $48 \mathrm{~h}$ of after transfection, the expression of miR-214 was measured by RT-qPCR. The results revealed that miR-214 expression was significantly increased in the MCF-7 cells transfected with the miR-214 mimic compared with those transfected with the mimic control (Fig. 2A), while it was significantly decreased in the miR-214 inhibitor-transfected group compared with the group transfected with the inhibitor control (Fig. 2B). The cell invasion ability was analyzed using Transwell cell culture chambers. The results revealed that the invasion ability of the MCF-7 cells was significantly increased in the cells transfected with the miR-214 mimic compared with those transfected with the mimic control (Fig. 2C), while the invasion ability was significantly decreased in the miR-214 inhibitor-transfected group compared with the group transfected with the inhibitor control (Fig. 2D).
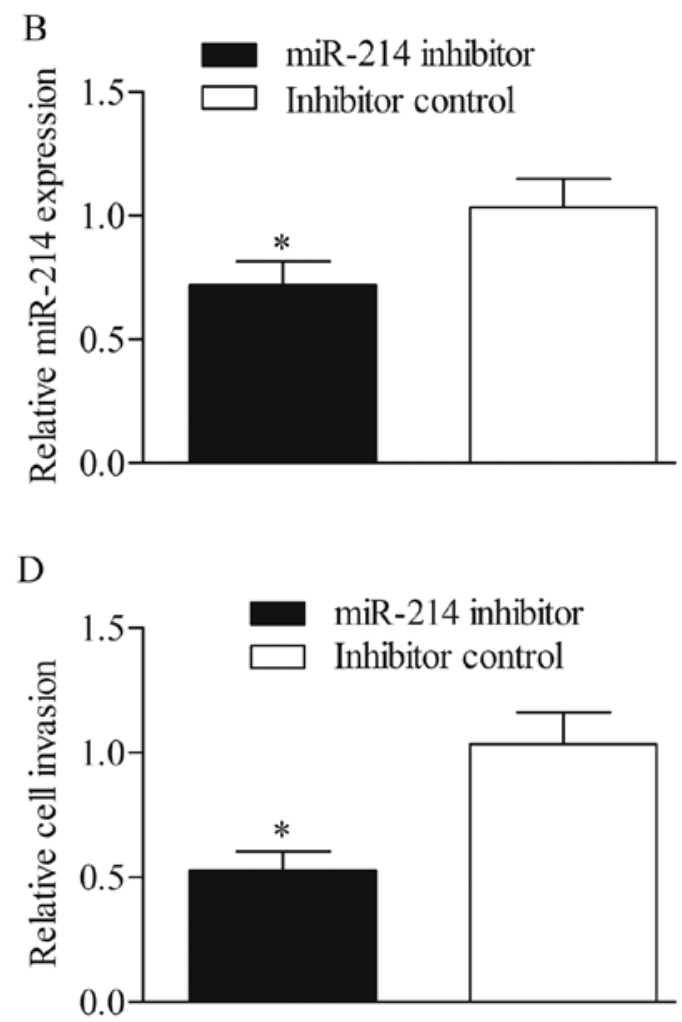

Figure 2. miR-214 regulates the invasion ability of MCF-7 cells. Detection of miR-214 expression levels in (A) miR-214 mimic- or (B) miR-214 inhibitortransfected groups by RT-qPCR. The miR-214 mimic, miR-214 inhibitor and corresponding controls were transfected into MCF-7 cells. Detection of the effects of (C) miR-214 mimic or (D) miR-214 inhibitor on the invasion ability of MCF-7 cells using Transwell cell culture chambers. Data are represented as the means $\pm \mathrm{SD}(\mathrm{n}=6)$. ${ }^{*} \mathrm{P}<0.05$ vs. mimic or inhibitor control group. 
A

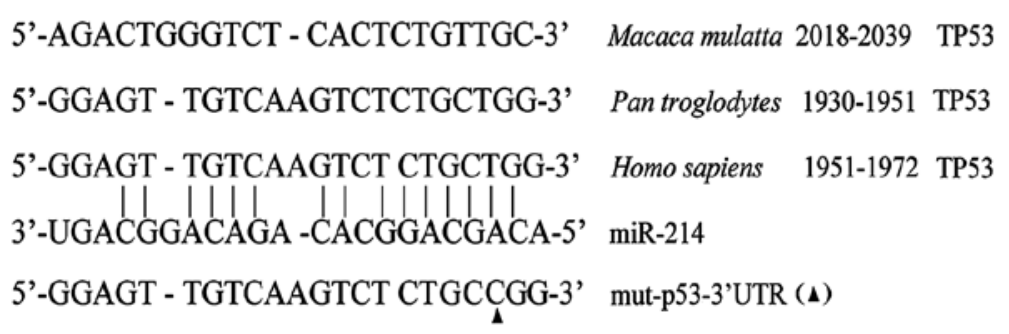

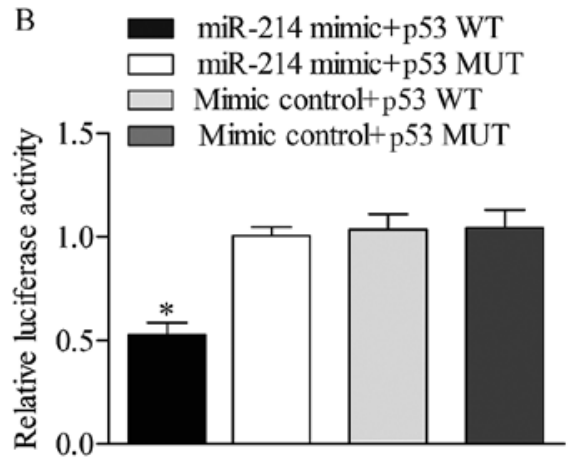

Figure 3. Identification of the targeting sequence between miR-214 and p53. The target gene was predicted using bioinformatics software programs and identified by luciferase reporter assay. (A) The wild-type (WT) miR-214 binding sequence and the mutant (MUT) miR-214 binding sequence in the 3 '-untranslated region (3'-UTR) of the p53 gene. (B) Relative firefly luciferase activity in MCF-7 cells co-transfected with miR-214 mimic or mimic control together with luciferase reporter constructs containing either WT or MUT p53 3'-UTR. Data are represented as the means \pm SD $(n=3)$. " $P<0.05$ vs. other 3 groups.
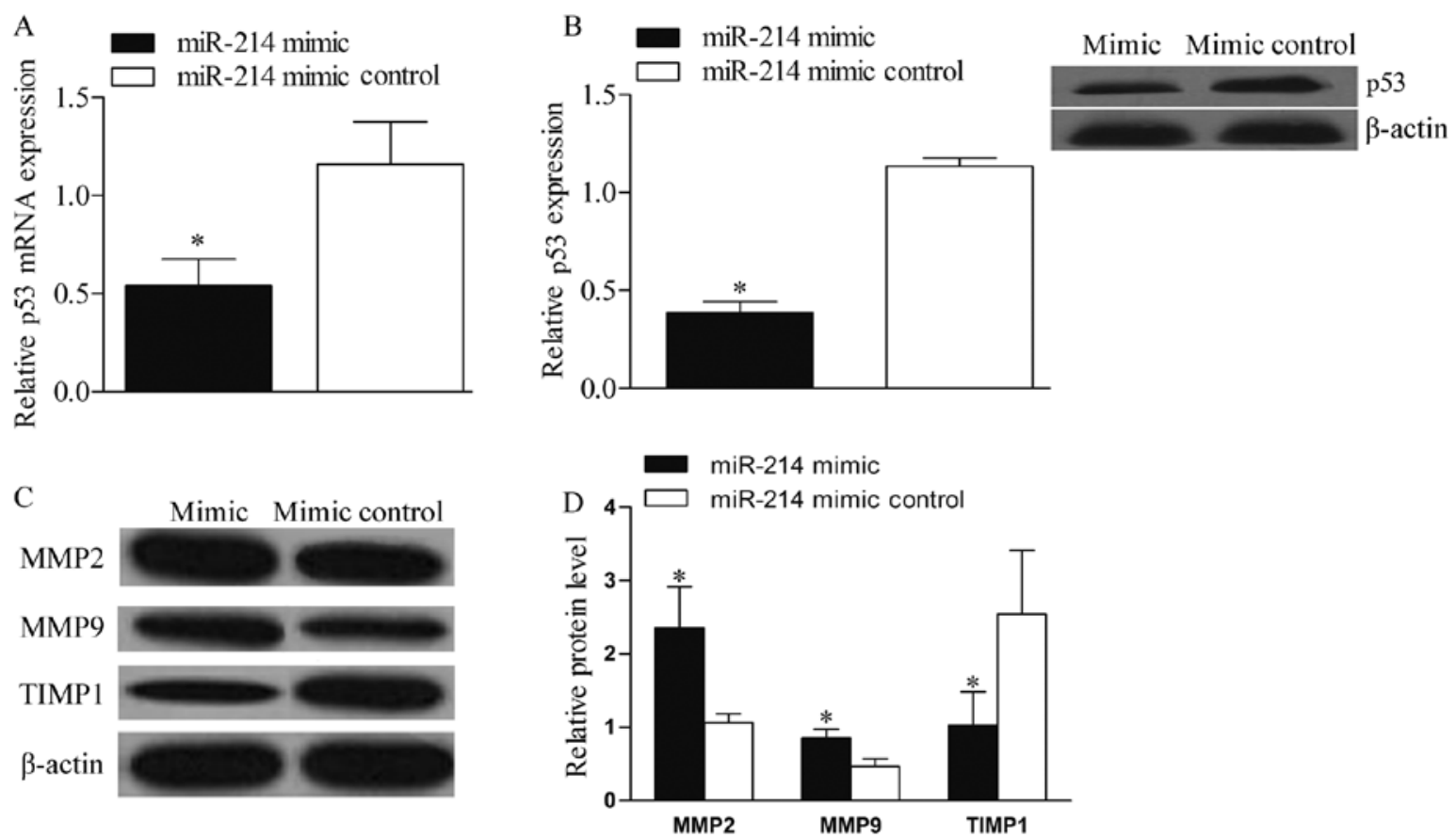

Figure 4. Effect of miR-214 overexpression on 553 expression and the protein expression levels of tissue inhibitor of matrix metalloproteinase 1 (TIMP1), matrix metalloproteinase (MMP)2 and MMP9 in breast cancer (BC) cells. miR-214 mimic or miR-214 mimic control was transfected into the MCF-7 cells. (A) The mRNA expression levels of $\mathrm{p} 53$ were measured by RT-qPCR. The protein levels of (B) p53 and (C) TIMP1, MMP2 and MMP9 were measured by western blot analysis. (D) Relative protein expression was quantified using Image-Pro Plus 6.0 software and normalized to $\beta$-actin. Data are represented as the means \pm SD $(\mathrm{n}=6) .{ }^{*} \mathrm{P}<0.05$ vs. mimic control.

Prediction and identification of the target gene of miR-214. The bioinformatics software programs, miRBase, RegRNA, TargetScan and RNA22, were used to predict the target gene of miR-214. According to the sequence analysis with RNA22, the potential binding target sequence of miR-214 was found in the 3 '-UTR of the p53 gene (Fig. 3A). The luciferase reporter vectors of p53 wild-type (WT) and p53 mutant type (MUT), which contained the WT and MUT of the potential binding sequence in the 3'-UTR of p53 respectively, were constructed and co-transfected with the miR-214 mimics or the miR-214 mimic control into the MCF-7 cells to determine whether $\mathrm{p} 53$ is a target of miR-214. At $48 \mathrm{~h}$ after transfection, the results revealed that the luciferase activity in the cells co-transfected with p53 WT and the miR-214 mimic was significantly reduced compared with the other 3 groups (Fig. 3B). However, there was no significant difference in luciferase activity among the groups of cells co-transfected with p53 WT and the miR-214 mimic control, p53 MUT and the miR-214 mimic and with p53 MUT and the miR-214 mimic control (Fig. 3B).

Overexpression of miR-214 inhibits p53 expression and upregulates the protein expression levels of MMP2 and MMP9, but downregulates the expression levels of TIMPI in BC cells. The miR-214 mimic and mimic control were transfected into the MCF-7 cells to determine the effects of miR-214 overexpression on BC cells. At $48 \mathrm{~h}$ after transfection, the mRNA and protein levels of p53 were measured by RT-qPCR and western blot analysis. The results revealed that the mRNA levels of p53 were significantly decreased in the MCF-7 cells transfected with the miR-214 mimic compared with those transfected 

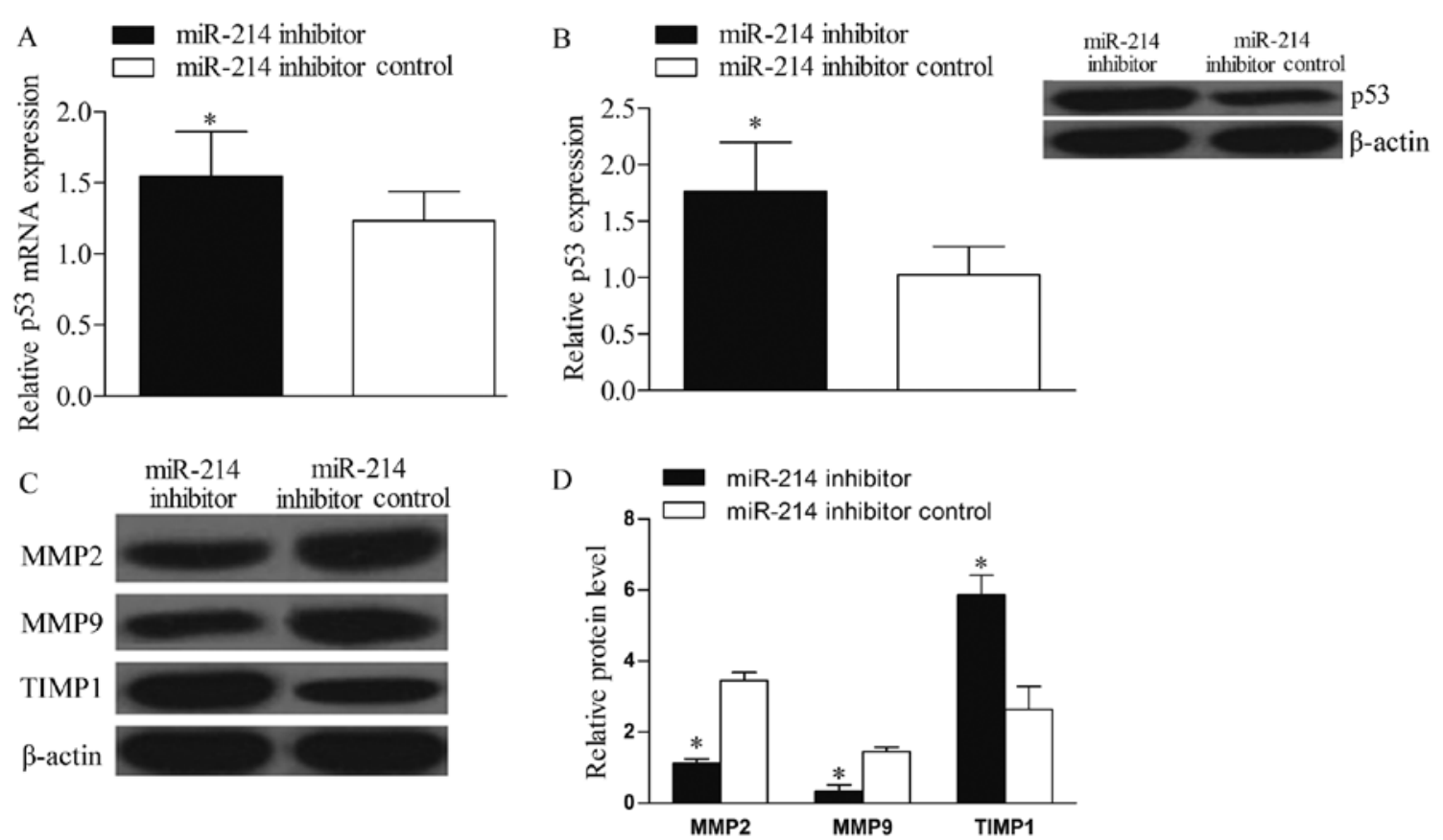

Figure 5. Effect of inhibition of miR-214 on p53 expression and protein expression levels of tissue inhibitor of matrix metalloproteinase 1 (TIMP1), matrix metalloproteinase (MMP)2 and MMP9 in breast cancer (BC) cells. miR-214 inhibitor or miR-214 inhibitor control were transfected into the MCF-7 cells. (A) The mRNA expression levels of p53 were measured by RT-qPCR. The protein levels of (B) p53 and (C) TIMP1, MMP2 and MMP9 were measured by western blot analysis. (D) Relative protein expression was quantified using Image-Pro Plus 6.0 software and normalized to $\beta$-actin. Data are represented as the means $\pm \mathrm{SD}(\mathrm{n}=6) .{ }^{*} \mathrm{P}<0.05$ vs. inhibitor control.

with the mimic control (Fig. 4A), which was consistent with the results of western blot analysis (Fig. 4B). Subsequently, the expression of proteins related to cell invasion (TIMP1, MMP2 and MMP9) was measured by western blot analysis. The results revealed that the expression levels of MMP2 and MMP9 were significantly increased, while the expression of TIMP1 was significantly decreased in the miR-214 mimictransfected group compared with the group transfected with the mimic control (Fig. 4C and D).

Inhibition of miR-214 increases p53 expression and downregulates the protein expression levels of MMP2 and MMP9, but upregulates TIMP1 expression in BC cells. The miR-214 inhibitor and inhibitor control were transfected into the MCF-7 cells to determine whether the inhibition of miR-214 reverses the enhancement of cell invasion. The results of RT-qPCR revealed that the mRNA expression level of p53 was significantly increased in the cells transfected with the miR-214 inhibitor compared with those transfected with the inhibitor control (Fig. 5A). The results of western blot analysis were similar; the protein expression level of p53 was significantly increased in the cells transfected with the miR-214 inhibitor (Fig. 5B). Additionally, the expression levels of MMP2 and MMP9 were significantly decreased, while the expression level of TIMP1 was significantly increased in the cells transfected with the miR-214 inhibitor compared with those transfected with the inhibitor control (Fig. 5C and D).

Overexpression of p53 abrogates miR-214-induced cell invasion in BC cells. In order to further verify whether miR-241 enhances the invasion ability of the MCF-7 cells through the regulation of p53 expression, the cells were co-transfected with the miR-214 mimic and p53 overexpression vector harboring no specific miR-214 binding-specific sequences in the 3'-UTR. The results revealed that the overexpression of p53 (Fig. 6A) significantly blocked the miR-214-induced invasion ability of the MCF-7 cells (Fig. 6B). Furthermore, the overexpression of p53 abrogated the increase in the expression of MMP2 and MMP9 (proteins related to cell invasion-related) and the decrease in TIMP1 expression (Fig. 6C and D). In conclusion, these findings suggest that miR-214 plays an important role in the regulation of the invasion ability of BC cells by targeting p53.

\section{Discussion}

$\mathrm{BC}$ is the foremost cause of cancer-related mortality in women worldwide, ranking as the second most common type of cancer, with 1.38 million new cases diagnosed and 458,100 deaths recorded in 2008 (25). The natural history of BC commonly involves the progression of in situ hyperproliferative lesions, invasive carcinomas and, eventually, metastatic disease (26). $\mathrm{BC}$ cell invasion is an important process in tumor metastasis, which is associated with various molecular alterations $(27,28)$. However, the detailed molecular mechanisms responsible for $\mathrm{BC}$ cell invasion remain unclear. Recently, miRNAs have emerged as an important mechanism responsible for $\mathrm{BC}$ cell invasion $(29,30)$. In this study, we demonstrated that the expression levels of miR-214 were upregulated in $\mathrm{BC}$ tissues compared with adjacent benign tissues. Moreover, we demonstrated that the dysregulation of miR-214 is involved in the mechanisms of BC cell invasion by targeting p53.

miRNAs are known to be endogenous single-stranded noncoding RNAs consisting of approximately 22 nucleotides, which can suppress the post-transcriptional expression of target genes 

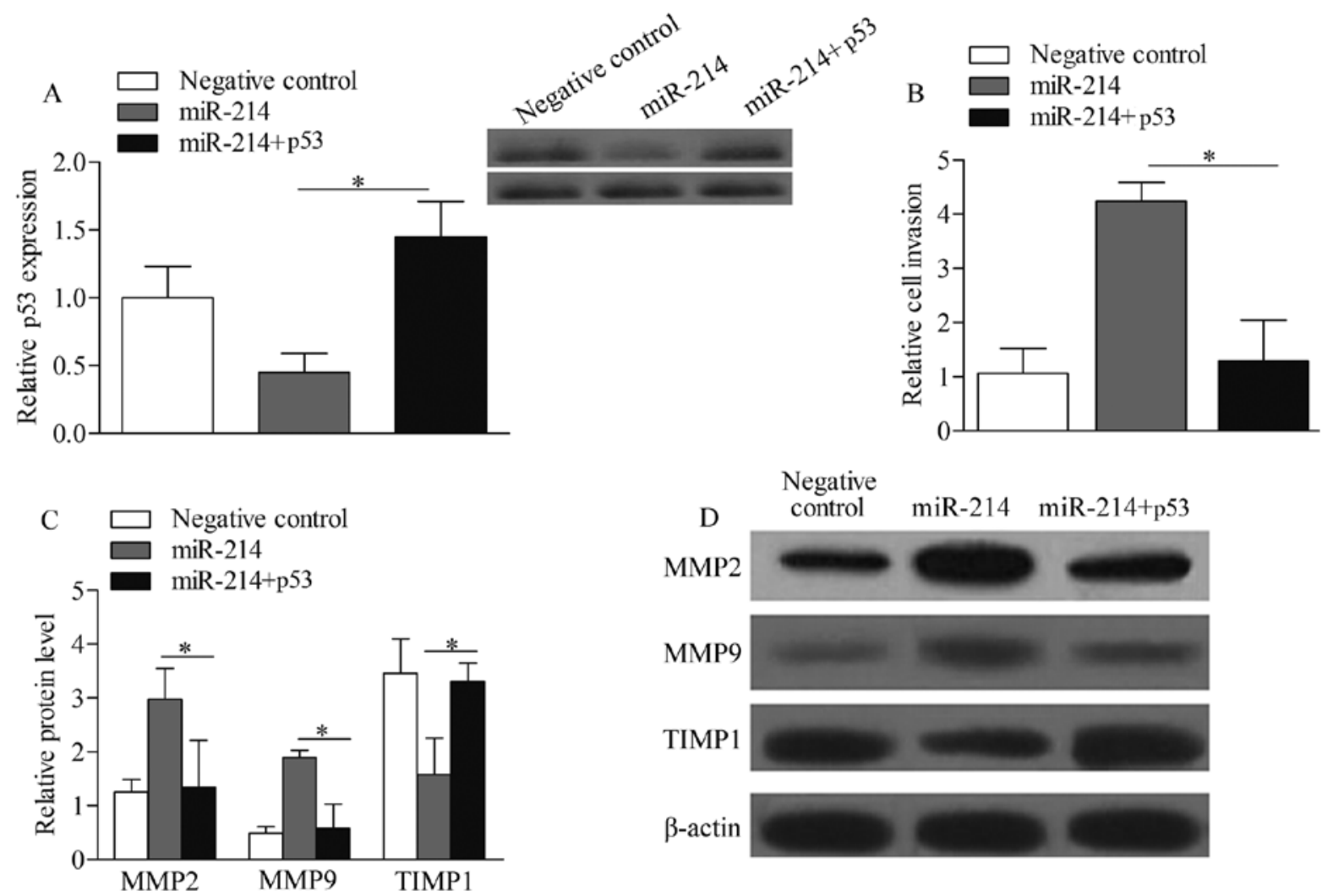

Figure 6. Effect of p53 overexpression on miR-214-induced enhancement of the invasion of breast carcinoma (BC) cells. miR-214 and p53 overexpression vectors harboring no miR-214 binding-specific sequences in the 3'-untranslated region (3'-UTR) were co-transfected into the MCF-7 cells. (A) The expression levels of p53 were detected by western blot analysis. (B) The invasion ability of MCF-7 cells was analyzed using Transwell cell culture chambers. (C) The expression levels of tissue inhibitor of matrix metalloproteinase 1 (TIMP1), matrix metalloproteinase (MMP)2 and MMP9 were detected by western blot analysis, and (D) were quantified using Image-Pro Plus 6.0 software and normalized to $\beta$-actin. Data are represented as the means $\pm \mathrm{SD}(\mathrm{n}=3)$. ${ }^{*} \mathrm{P}<0.05 \mathrm{miR}-214$.

and have been demonstrated to promote or suppress different cellular processes, e.g., cell apoptosis, differentiation, development, metastasis and invasion (31-34). An increasing number of studies have reinforced the notion that miRNAs are frequently deregulated and aberrantly expressed in certain types of cancer. Among these, miR-214 has been shown to play a role in the development of several types of cancer $(15,16)$. In a previous study, miR-214 was observed to be upregulated in BC (24). In the present study, the expression levels of miR-214 were significantly elevated in BC tissues in contrast to adjacent benign tissues, which was consistent with the results of this former study (24). Furthermore, in our study, we detected a strong correlation between the upregulation of miR-214 and the invasion ability of $\mathrm{BC}$ cells. These results demonstrate that miR-214 functions as an oncogene to regulate the invasion ability of BC cells.

A recent study demonstrated that $\mathrm{p} 53$ was the target gene of miR-214 (35). In the present study, a conservative miR-214 binding site in the 3'-UTR of p53 was detected using bioinformatics software programs. Moreover, the results of luciferase reporter assay revealed that the overexpression of miR-214 reduced the luciferase activity when the cells were transfected with p53 WT, but not when transfected with p53 MUT. These results demonstrate that p53 is a direct target of miR-214.p53 is a well known tumor suppressor gene in various types of cancer. Its critical function in tumorigenesis is reflected by the fact that the gene is directly mutated or silenced in almost half of all human malignant tumors $(36,37)$. The p53 tumor suppressor gene is primarily known for its important role in the maintenance of genome integrity by controlling the cell cycle, apoptosis and DNA repair (38). Recently, Lam et al (39) reported that p53 impedes tumor cell invasion and migration in breast cancer. Thus, in order to investigate the role of miR-214 in cell invasion by targeting p53, we determined the effects of miR-214 overexpression on the expression of p53 and the invasion in MCF-7 cells. The results revealed that p53 was significantly decreased due to the overexpression of miR-214. It has also been reported that MMPs, such as MMP2 and MMP9, participate in the degradation of the extracellular matrix (ECM), leading to tumor cell invasion (40). Additionally, MMPs are negatively regulated by TIMPs (41). In the present study, we detected the expression levels of proteins related to cell invasion (TIMP1, MMP2 and MMP9) in miR-214 mimic-transfected MCF-7 cells. The results revealed that the overexpression of miR-214 significantly increased the expression of MMP2 and MMP9, while it decreased the expression of TIMP1. Taken together, these results suggest that the overexpression of miR-214 induces the invasion ability of BC cells by decreasing p53 expression, which may lead to the progression of BC.

A recent study discovered several miRNAs that are potential therapeutic targets for BC (42). In this study, in order to investigate the therapeutic potential of miR-214 in $\mathrm{BC}$, we detected the effects of miR-214 inhibition on the expression 
of p53 and the invasion ability of MCF-7 cells. The results revealed that $\mathrm{p} 53$ expression was significantly increased due to the inhibition of miR-214. Moreover, the expression of MMP2 and MMP9 was significantly decreased, while that of TIMP9 was significantly increased (proteins related to cell invasion) following transfection of the cells with miR-214 inhibitor. These results suggest that miR-214 regulates $\mathrm{BC}$ cell invasion by targeting p53. Furthermore, we co-transfected the cells with miR-214 mimic and p53 overexpression vector, which harbored no specific miR-214 binding-specific sequences in the 3'-UTR, and detected the effects of the overexpression of p53 on cell invasion. The results revealed that the overexpression of $\mathrm{p} 53$ significantly inhibited the miR-214-induced enhancement of the invasion of MCF-7 cells and verified that miR-214 enhances the invasion ability of $\mathrm{BC}$ cells by targeting p53.

In conclusion, our results demonstrate that the upregulation of miR-214 affects the expression of p53, leading to the enhancement of BC cell invasion ability, which ultimately facilitates the development of BC. Consequently, this study provides important insight into the pathogenesis of $\mathrm{BC}$ cell invasion and implicates miR-214 as a potential therapeutic target in the treatment of $\mathrm{BC}$.

\section{Acknowledgements}

The authors would like to thank the members of the Second Department of Breast Surgery, The First Affiliated Hospital of Zhengzhou University, for providing helpful discussions concerning the present study.

\section{References}

1. Motamedolshariati M, Memar B, Aliakbaian M, Shakeri MT, Samadi M and Jangjoo A: Accuracy of prognostic and predictive markers in core needle breast biopsies compared with excisional specimens. Breast Care Basel 9: 107-110, 2014.

2. Youlden DR, Cramb SM, Dunn NA, Muller JM, Pyke CM and Baade PD: The descriptive epidemiology of female breast cancer: An international comparison of screening, incidence, survival and mortality. Cancer Epidemiol 36: 237-248, 2012.

3. Bartel DP: MicroRNAs: Target recognition and regulatory functions. Cell 136: 215-233, 2009.

4. Thorn SR, Regnault TR, Brown LD, Rozance PJ, Keng J, Roper M, Wilkening RB, Hay WW Jr and Friedman JE: Intrauterine growth restriction increases fetal hepatic gluconeogenic capacity and reduces messenger ribonucleic acid translation initiation and nutrient sensing in fetal liver and skeletal muscle. Endocrinology 150: 3021-3030, 2009.

5. Cheng H, Zhang L, Cogdell DE, Zheng H, Schetter AJ, Nykter M, Harris CC, Chen K, Hamilton SR and Zhang W: Circulating plasma MiR-141 is a novel biomarker for metastatic colon cancer and predicts poor prognosis. PLoS One 6: e17745, 2011.

6. Fornari F, Milazzo M, Chieco P, et al: In hepatocellular carcinoma miR-519d is up-regulated by 533 and DNA hypomethylation and targets CDKN1A/p21, PTEN, AKT3 and TIMP2. J Pathol 227: 275-285, 2012.

7. Farazi TA, Hoell JI, Morozov P and Tuschl T: MicroRNAs in human cancer. In: MicroRNA Cancer Regulation. Springer, pp1-20, 2013

8. Volinia S, Galasso M, Sana ME, Wise TF, Palatini J, Huebner K and Croce CM: Breast cancer signatures for invasiveness and prognosis defined by deep sequencing of microRNA. Proc Natl Acad Sci USA 109: 3024-3029, 2012.

9. Wang D, Qiu C, Zhang H, Wang J, Cui Q and Yin Y: Human microRNA oncogenes and tumor suppressors show significantly different biological patterns: From functions to targets. PLoS One 5: 5, 2010

10. Tang J, Ahmad A and Sarkar FH: The role of microRNAs in breast cancer migration, invasion and metastasis. Int J Mol Sci 13: 13414-13437, 2012.
11. He ML, Luo MX, Lin MC and Kung HF: MicroRNAs: Potential diagnostic markers and therapeutic targets for EBV-associated nasopharyngeal carcinoma. Biochim Biophys Acta 1825: 1-10, 2012.

12. Zhang J, Fei B, Wang Q, et al: MicroRNA-638 inhibits cell proliferation, invasion and regulates cell cycle by targeting tetraspanin 1 in human colorectal carcinoma. Oncotarget 5: 12083-12096, 2014.

13. Wang L, Gao W, Hu F, Xu Z and Wang F: MicroRNA-874 inhibits cell proliferation and induces apoptosis in human breast cancer by targeting CDK9. FEBS Lett 588: 4527-4535, 2014.

14. Zhou SW, Su BB, Zhou Y, Feng YQ, Guo Y, Wang YX, Qi P and $\mathrm{Xu}$ S: Aberrant miR-215 expression is associated with clinical outcome in breast cancer patients. Med Oncol 31: 259, 2014.

15. Penna E, Orso F, Cimino D, et al: microRNA-214 contributes to melanoma tumour progression through suppression of TFAP2C. EMBO J 30: 1990-2007, 2011.

16. Shih TC, Tien YJ, Wen CJ, Yeh TS, Yu MC, Huang $\mathrm{CH}$, Lee YS, Yen TC and Hsieh SY: MicroRNA-214 downregulation contributes to tumor angiogenesis by inducing secretion of the hepatoma-derived growth factor in human hepatoma. J Hepatol 57: 584-591, 2012.

17. Wang Z, Cai H, Lin L, Tang M and Cai H: Upregulated expression of microRNA-214 is linked to tumor progression and adverse prognosis in pediatric osteosarcoma. Pediatr Blood Cancer 61: 206-210, 2014.

18. Yin G, Chen R, Alvero AB, Fu HH, Holmberg J, Glackin C, Rutherford T and Mor G: TWISTing stemness, inflammation and proliferation of epithelial ovarian cancer cells through MIR199A2/214. Oncogene 29: 3545-3553, 2010.

19. Ueda T, Volinia S, Okumura H, et al: Relation between microRNA expression and progression and prognosis of gastric cancer: A microRNA expression analysis. Lancet Oncol 11: 136-146, 2010

20. Narducci MG, Arcelli D, Picchio MC, et al: MicroRNA profiling reveals that miR-21, miR486 and miR-214 are upregulated and involved in cell survival in Sézary syndrome. Cell Death Dis 2: e151, 2011.

21. Yang Z, Chen S, Luan X, Li Y, Liu M, Li X, Liu T and Tang H: MicroRNA-214 is aberrantly expressed in cervical cancers and inhibits the growth of HeLa cells. IUBMB Life 61: 1075-1082, 2009.

22. Zhang XJ, Ye H, Zeng CW, He B, Zhang H and Chen YQ: Dysregulation of miR-15a and miR-214 in human pancreatic cancer. J Hematol Oncol 3: 46, 2010.

23. Duan Q, Wang X, Gong W, Ni L, Chen C, He X, Chen F, Yang L, Wang P and Wang DW: ER stress negatively modulates the expression of the miR-199a/214 cluster to regulates tumor survival and progression in human hepatocellular cancer. PLoS One 7: e31518, 2012

24. Schwarzenbach H, Milde-Langosch K, Steinbach B, Müller V and Pantel K: Diagnostic potential of PTEN-targeting miR-214 in the blood of breast cancer patients. Breast Cancer Res Treat 134: 933-941, 2012.

25. Jemal A, Bray F, Center MM, Ferlay J, Ward E and Forman D: Global cancer statistics. CA Cancer J Clin 61: 69-90, 2011.

26. Hu M, Yao J, Carroll DK, et al: Regulation of in situ to invasive breast carcinoma transition. Cancer Cell 13: 394-406, 2008.

27. Furlan A, Vercamer C, Bouali F, Damour I, Chotteau-Lelievre A, Wernert N, Desbiens X and Pourtier A: Ets-1 controls breast cancer cell balance between invasion and growth. Int J Cancer 135: 2317-2328, 2014.

28. Jaafar H, Sharif SE and Murtey MD: Pattern of collagen fibers and localization of matrix metalloproteinase 2 and 9 during breast cancer invasion. Tumori 100: e204-e211, 2014.

29. Ye ZB, Ma G, Zhao YH, et al: miR-429 inhibits migration and invasion of breast cancer cells in vitro. Int J Oncol 46: 531-538, 2015.

30. Meng Y,Zou Q, Liu T, Cai X, Huang Y and Pan J: microRNA-335 inhibits proliferation, cell-cycle progression, colony formation, and invasion via targeting PAX6 in breast cancer cells. Mol Med Rep 11: 379-385, 2015.

31. Hidaka H, Yoshino H, Enokida H, et al: Tumor-suppressive miR-135a inhibits cancer cell proliferation by targeting the c-MYC oncogene in renal cell carcinoma. J Urol 189: e190, 2013.

32. Baumjohann D, Kageyama R, Clingan JM, et al: 16: MIR-17 92 promotes $\mathrm{T}$ follicular helper cell differentiation and represses subset-inappropriate gene expression. Cytokine 63: 246-247, 2013.

33. Zhou Y, Xiong M, Niu J, Sun Q, Su W, Zen K, Dai C and Yang J: Secreted fibroblast-derived miR-34a induces tubular cell apoptosis in fibrotic kidney. J Cell Sci 127: 4494-4506, 2014. 
34. Zhao M, Huang J, Gui K, Xiong M, Cai G, Xu J, Wang K, Liu D, Zhang X and Yin W: The downregulation of miR-144 is associated with the growth and invasion of osteosarcoma cells through the regulation of TAGLN expression. Int J Mol Med 34: 1565-1572, 2014.

35. Misiewicz-Krzeminska I, Sarasquete ME, Quwaider D, et al: Restoration of microRNA-214 expression reduces growth of myeloma cells through positive regulation of P53 and inhibition of DNA replication. Haematologica 98: 640-648, 2013.

36. Walerych D, Napoli M, Collavin L and Del Sal G: The rebel angel: Mutant p53 as the driving oncogene in breast cancer. Carcinogenesis 33: 2007-2017, 2012.

37. Olivier M, Hollstein M and Hainaut P: TP53 mutations in human cancers: Origins, consequences, and clinical use. Cold Spring Harb Perspect Biol 2: a001008, 2010.

38. Lane D and Levine A: p53 Research: The past thirty years and the next thirty years. Cold Spring Harb Perspect Biol 2: a000893, 2010 .
39. Lam S, Wiercinska E, Teunisse AF, Lodder K, ten Dijke P and Jochemsen AG: Wild-type p53 inhibits pro-invasive properties of TGF- $\beta 3$ in breast cancer, in part through regulation of EPHB2, a new TGF- $\beta$ target gene. Breast Cancer Res Treat 148: 7-18, 2014.

40. Noh S, Jung JJ, Jung M, Kim KH, Lee HY, Wang B, Cho J, Kim TS, Jeung HC and Rha SY: Body fluid MMP-2 as a putative biomarker in metastatic breast cancer. Oncol Lett 3: 699-703, 2012.

41. Wang J, Gao Y, Ma M, Li M, Zou D, Yang J, Zhu Z and Zhao X: Effect of miR-21 on renal fibrosis by regulating MMP-9 and TIMP1 in kk-ay diabetic nephropathy mice. Cell Biochem Biophys 67: 537-546, 2013.

42. Piva R, Spandidos DA and Gambari R: From microRNA functions to microRNA therapeutics: Novel targets and novel drugs in breast cancer research and treatment (Review). Int J Oncol 43: 985-994, 2013. 